Acta Crystallographica Section E

Structure Reports

Online

ISSN 1600-5368

\section{Elaidic acid (trans-9-octadecenoic acid)}

\section{John Nicolson Low, ${ }^{\mathrm{a} *}$ Charlie Scrimgeour $^{b}$ and Peter Horton ${ }^{c}$}

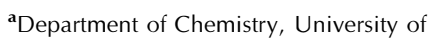
Aberdeen, Meston Walk, Old Aberdeen AB24 3UE, Scotland, ${ }^{\mathbf{b}}$ Scottish Crop Research Institute, Invergowrie, Dundee DD2 5DA, Scotland, and ${ }^{\mathrm{C}}$ EPSRC National Crystallography Service, School of Chemistry, University of Southampton, Highfield, Southampton SO17 1BJ, England

Correspondence e-mail: che562@abdn.ac.uk

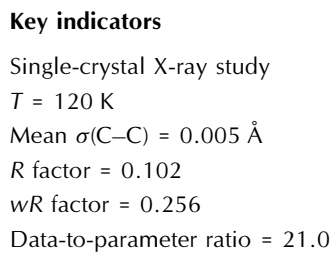

Key indicators

Single-crystal X-ray study

$T=120 \mathrm{~K}$

Mean $\sigma(\mathrm{C}-\mathrm{C})=0.005 \AA$

$R$ factor $=0.102$

$w R$ factor $=0.256$

Data-to-parameter ratio $=21.0$

For details of how these key indicators were automatically derived from the article, see http://journals.iucr.org/e.
Elaidic acid, $\mathrm{C}_{18} \mathrm{H}_{34} \mathrm{O}_{2}$, has an essentially linear alkyl chain. The double bond is twisted across the mean direction of the alkyl chain in a skew', trans, skew conformation. In the crystal structure, the molecules form centrosymmetric $\mathrm{O}-\mathrm{H} \cdots \mathrm{O}$ hydrogen-bonded dimers $(\mathrm{O} \cdots \mathrm{O}=2.684 \AA$ ) .

\section{Comment}

The physical, biological and nutritional properties of fatty acids are largely determined by the number, position and configuration of their double bonds. These determine the shape of the molecules, the way molecules can pack together in solid phases, monolayers, bilayers etc., and how individual molecules can interact with enzymes and receptors. Most natural unsaturated fatty acids have cis $(Z)$ double bonds. Trans $(E)$ fatty acids are present in dairy fats and are produced during the catalytic partial hydrogenation used in the production of hardened fats and during deodorization of commodity oils. The labelling of foods with trans content is increasingly required due to their undesirable nutritional properties. Alternative ways of producing hardened fats, such as interesterification or blending with fully saturated fats, and milder deodorization procedures, are being developed to reduce trans content. Trans fatty acids more closely resemble saturated acids in melting point and nutritional properties, sharing an essentially linear structure which allows closely aligned packing in condensed phases. In contrast, cis double bonds introduce a bend in the alkyl chain, making packing less stable and lowering the melting point. We have determined the structure of elaidic acid (trans-9-octadecenoic acid), (I), to enable a detailed comparison of a trans fatty acid with saturated and cis-unsaturated compounds.

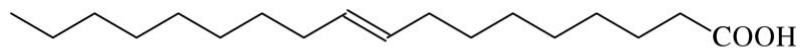

(I)

Relatively few crystal structures of fatty acids are available, as good crystals are difficult to obtain, often being thin plates and often crystallizing in several polymorphs. Most monoenes have low melting points and polyenes are liquids at room temperature. The crystal structures of the following saturated and monoene $\mathrm{C}_{18}$ fatty acids have been reported to date: stearic acid (octadecanoic acid) (Malta et al., 1971; Kaneko et al., 1990, 1994a,b), oleic acid (cis-9-octadecenoic acid) (Abrahamsson \& Ryderstedt-Nahringbauer, 1962; Kaneko et al., 1997) and petroselinic acid (cis-6-octadecenoic acid) (Kaneko et al., 1992a,b). No trans-octadecenoic acid structure has been reported to date.
Received 11 October 2005 Accepted 14 October 2005 Online 19 October 2005
(C) 2005 International Union of Crystallography Printed in Great Britain - all rights reserved 


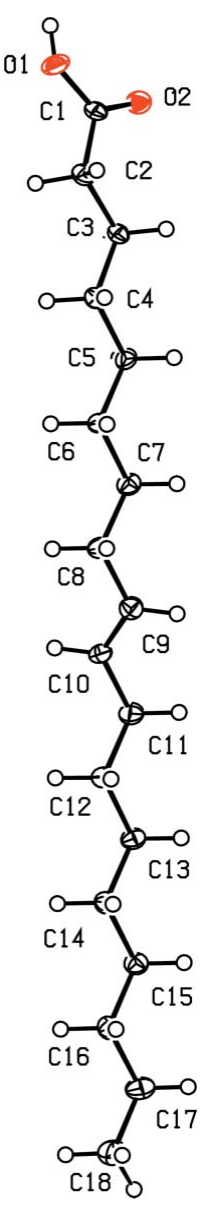

Figure 1

A view of (I), with displacement ellipsoids drawn at the $30 \%$ probability level.

Elaidic acid (I) has an essentially linear alkyl chain, with the torsion angle between saturated $\mathrm{C}$ atoms close to $180^{\circ}$ (Table 1). The $\mathrm{C} 7-\mathrm{C} 8-\mathrm{C} 9-\mathrm{C} 10, \mathrm{C} 8-\mathrm{C} 9-\mathrm{C} 10-\mathrm{C} 11$ and $\mathrm{C} 9-\mathrm{C} 10-\mathrm{C} 11-\mathrm{C} 12$ torsion angles are $-118.8(4)$, -179.9 (4) and $118.6(4)^{\circ}$, respectively, resulting in the double bond being twisted across the mean direction of the alkyl chain in a skew', trans, skew conformation. The C1-C18 distance is $21.393(6) \AA$, comparable with that in fully extended stearic acid structures (21.6 ̊; Malta et al., 1971; Kaneko et al., 1990, 1994b). This contrasts with the cis-octadecenoic acids, where the molecules are bent and the C1-C18 distance is reduced to between 17.8 and $19.7 \AA$ (Abrahamsson \& Ryderstedt-Nahringbauer, 1962; Kaneko et al., 1997, 1992a,b).

In the crystal structure of (I), molecules related by inversion centres are linked by $\mathrm{O}-\mathrm{H} \cdots \mathrm{O}$ hydrogen bonds to form $R_{2}^{2}(8)$ dimers (Bernstein et al., 1995) typical of carboxylic acids (Table 2).

\section{Experimental}

A commercial sample of (I) (Sigma, Poole, Dorset, UK) was recrystallized from ethanol at room temperature. The crystals were composed of very thin stacked sheets which tended to be twisted. After many attempts, a crystal was found from which it was possible to obtain a data set.
Crystal data

\begin{tabular}{|c|c|}
\hline $\mathrm{C}_{18} \mathrm{H}_{34} \mathrm{O}_{2}$ & $D_{x}=1.075 \mathrm{Mg} \mathrm{m}^{-3}$ \\
\hline$M_{r}=282.45$ & Mo $K \alpha$ radiation \\
\hline $\begin{array}{l}\text { Monoclinic, } C 2 / c \\
a=98.48(2) \AA\end{array}$ & $\begin{array}{l}\text { Cell parameters from } 3830 \\
\text { reflections }\end{array}$ \\
\hline$b=4.9381(3) \AA$ & $\theta=3.3-27.6^{\circ}$ \\
\hline$c=7.1826(8) \AA$ & $\mu=0.07 \mathrm{~mm}^{-1}$ \\
\hline$\beta=92.570(12)^{\circ}$ & $T=120(2) \mathrm{K}$ \\
\hline$V=3489.4(8) \AA^{3}$ & Plate, colourless \\
\hline$Z=8$ & $0.20 \times 0.18 \times 0.01 \mathrm{~mm}$ \\
\hline
\end{tabular}

Data collection

Bruker Nonius KappaCCD areadetector diffractometer

$\varphi$ and $\omega$ scans

Absorption correction: multi-scan (SADABS; Sheldrick, 2003)

$T_{\min }=0.987, T_{\max }=0.999$

17532 measured reflections

Refinement

Refinement on $F^{2}$

$R\left[F^{2}>2 \sigma\left(F^{2}\right)\right]=0.102$

$w R\left(F^{2}\right)=0.256$

$S=1.08$

3830 reflections

182 parameters

$\mathrm{H}$-atom parameters constrained

3830 independent reflections 1679 reflections with $I>2 \sigma(I)$

$R_{\text {int }}=0.150$

$\theta_{\text {max }}=27.6^{\circ}$

$h=-126 \rightarrow 126$

$k=-6 \rightarrow 6$

$l=-9 \rightarrow 9$

$$
\begin{gathered}
w=1 /\left[\sigma^{2}\left(F_{\mathrm{o}}^{2}\right)+(0.0744 P)^{2}\right. \\
\quad+7.8445 P] \\
\text { where } P=\left(F_{\mathrm{o}}{ }^{2}+2 F_{\mathrm{c}}^{2}\right) / 3 \\
(\Delta / \sigma)_{\max }=0.001 \\
\Delta \rho_{\max }=0.41 \mathrm{e}^{-3} \\
\Delta \rho_{\min }=-0.35 \mathrm{e}^{-3}
\end{gathered}
$$

Table 1

Selected torsion angles $\left({ }^{\circ}\right)$.

\begin{tabular}{lrlr}
\hline $\mathrm{C} 1-\mathrm{C} 2-\mathrm{C} 3-\mathrm{C} 4$ & $-170.7(3)$ & $\mathrm{C} 9-\mathrm{C} 10-\mathrm{C} 11-\mathrm{C} 12$ & $118.6(4)$ \\
$\mathrm{C} 2-\mathrm{C} 3-\mathrm{C} 4-\mathrm{C} 5$ & $177.0(3)$ & $\mathrm{C} 10-\mathrm{C} 11-\mathrm{C} 12-\mathrm{C} 13$ & $178.4(3)$ \\
$\mathrm{C} 3-\mathrm{C} 4-\mathrm{C} 5-\mathrm{C} 6$ & $-176.6(3)$ & $\mathrm{C} 11-\mathrm{C} 12-\mathrm{C} 13-\mathrm{C} 14$ & $-179.8(3)$ \\
$\mathrm{C} 4-\mathrm{C} 5-\mathrm{C} 6-\mathrm{C} 7$ & $178.9(3)$ & $\mathrm{C} 12-\mathrm{C} 13-\mathrm{C} 14-\mathrm{C} 15$ & $179.9(3)$ \\
$\mathrm{C} 5-\mathrm{C} 6-\mathrm{C} 7-\mathrm{C} 8$ & $-179.1(3)$ & $\mathrm{C} 13-\mathrm{C} 14-\mathrm{C} 15-\mathrm{C} 16$ & $179.8(3)$ \\
$\mathrm{C} 6-\mathrm{C} 7-\mathrm{C} 8-\mathrm{C} 9$ & $-178.4(3)$ & $\mathrm{C} 14-\mathrm{C} 15-\mathrm{C} 16-\mathrm{C} 17$ & $-179.6(3)$ \\
$\mathrm{C} 7-\mathrm{C} 8-\mathrm{C} 9-\mathrm{C} 10$ & $-118.8(4)$ & $\mathrm{C} 15-\mathrm{C} 16-\mathrm{C} 17-\mathrm{C} 18$ & $179.5(3)$ \\
$\mathrm{C} 8-\mathrm{C} 9-\mathrm{C} 10-\mathrm{C} 11$ & $-179.9(4)$ & & \\
\hline
\end{tabular}

Table 2

Hydrogen-bond geometry $\left(\AA{ }^{\circ}\right)$.

\begin{tabular}{lllll}
\hline$D-\mathrm{H} \cdots A$ & $D-\mathrm{H}$ & $\mathrm{H} \cdots A$ & $D \cdots A$ & $D-\mathrm{H} \cdots A$ \\
\hline $\mathrm{O} 1-\mathrm{H} 1 \cdots \mathrm{O}^{\mathrm{i}}$ & 0.87 & 1.86 & $2.684(3)$ & 158 \\
\hline
\end{tabular}

Symmetry code: (i) $-x+\frac{3}{2},-y+\frac{3}{2},-z+1$.

$\mathrm{H}$ atoms were treated as riding, with $\mathrm{C}-\mathrm{H}($ aromatic $)=0.95$ and $\mathrm{C}-\mathrm{H}\left(\mathrm{CH}_{2}\right)=0.99 \AA$, with $U_{\text {iso }}(\mathrm{H})=1.2 U_{\text {eq }}(\mathrm{C}), C-H($ methyl $)=$ $0.98 \AA$, with $U_{\text {iso }}(\mathrm{H})=1.5 U_{\text {eq }}(\mathrm{C})$, and $\mathrm{O}-\mathrm{H}=0.87 \AA$, with $U_{\text {iso }}(\mathrm{H})=$ $1.5 U_{\text {eq }}(\mathrm{O})$. The $\mathrm{O}$-bound $\mathrm{H}$ atom was allowed to ride at its position as determined from a difference map. Although the best crystal was selected from many crystallization attempts, the higher than usual values for $R, w R$ and $R_{\text {int }}$ may be a result of the crystal quality. The possibilty that the crystal was twinned was investigated but this did not give any significant results.

Data collection: COLLECT (Nonius, 1998); cell refinement: $D E N Z O$ (Otwinowski \& Minor, 1997) and COLLECT; data reduction: DENZO and COLLECT; program(s) used to solve structure: OSCAIL (McArdle, 2003) and SHELXS97 (Sheldrick, 1997); program(s) used to refine structure: OSCAIL and SHELXL97 (Sheldrick, 1997); molecular graphics: ORTEPII (Johnson, 1976) and PLATON (Spek, 2003); software used to prepare material for publication: SHELXL97 and PRPKAPPA (Ferguson, 1999). 


\section{organic papers}

The X-ray data were collected at the EPSRC X-ray Crystallographic Service, University of Southampton, England. The Scottish Crop Research Institute receives grant-in-aid from the Scottish Executive Environmental and Rural Affairs Department.

\section{References}

Abrahamsson, S. \& Ryderstedt-Nahringbauer, I. (1962). Acta Cryst. 15, 12611268.

Bernstein, J., Davis, R. E., Shimoni, L. \& Chang, N.-L. (1995). Angew. Chem. Int. Ed. Engl. 34, 1555-1573.

Ferguson, G. (1999). PRPKAPPA. University of Guelph, Canada.

Johnson, C. K. (1976). ORTEPII. Report ORNL-5138. Oak Ridge National Laboratory, Tennessee, USA.

Kaneko, F., Kobayashi, M., Kitagawa, Y. \& Matsuura, Y. (1990). Acta Cryst. C46, 1490-1492.

Kaneko, F., Kobayashi, M., Kitagawa, Y., Matsuura, Y., Sato, K. \& Suzuki, M. (1992a). Acta Cryst. C48, 1054-1057.
Kaneko, F., Kobayashi, M., Kitagawa, Y., Matsuura, Y., Sato, K. \& Suzuki, M. (1992b). Acta Cryst. C48, 1057-1060.

Kaneko, F., Sakashita, H., Kobayashi, M., Kitagawa, Y., Matsuura, Y. \& Suzuki, M. (1994a). Acta Cryst. C50, 245-247.

Kaneko, F., Sakashita, H., Kobayashi, M., Kitagawa, Y., Matsuura, Y. \& Suzuki, M. (1994b). Acta Cryst. C50, 247-250.

Kaneko, F., Yamazaki, K., Kitagawa, K., Kikyo, T., Kobayashi, M., Kitagawa, Y., Matsuura, Y., Sato, K. \& Suzuki, M. (1997). J. Phys. Chem. B, 101, $1803-$ 1809.

Malta, V., Celotti G., Zannetti, R. \& Martelli, A. F. (1971). J. Chem. Soc. B, pp. $548-553$.

McArdle, P. (2003). OSCAIL for Windows. Version 10. Crystallography Centre, Chemistry Department, NUI Galway, Ireland.

Nonius (1998). COLLECT. Nonius BV, Delft, The Netherlands.

Otwinowski, Z. \& Minor, W. (1997). Methods in Enzymology, Vol. 276, Macromolecular Crystallography, Part A, edited by C. W. Carter Jr \& R. M. Sweet, pp. 307-326. New York: Academic Press.

Sheldrick, G. M. (1997). SHELXS97 and SHELXL97. University of Göttingen, Germany.

Sheldrick, G. M. (2003). SADABS. Version 2.10. University of Göttingen, Germany.

Spek, A. L. (2003). J. Appl. Cryst. 36, 7-13. 


\section{supporting information}

Acta Cryst. (2005). E61, o3730-o3732 [https://doi.org/10.1107/S1600536805033040]

Elaidic acid (trans-9-octadecenoic acid)

John Nicolson Low, Charlie Scrimgeour and Peter Horton

trans-9-octadecenoic acid

Crystal data

$\mathrm{C}_{18} \mathrm{H}_{34} \mathrm{O}_{2}$

$M_{r}=282.45$

Monoclinic, $C 2 / c$

Hall symbol: $-\mathrm{C} 2 \mathrm{yc}$

$a=98.48(2) \AA$

$b=4.9381(3) \AA$

$c=7.1826(8) \AA$

$\beta=92.570(12)^{\circ}$

$V=3489.4(8) \AA^{3}$

$Z=8$

$F(000)=1264$

$D_{\mathrm{x}}=1.075 \mathrm{Mg} \mathrm{m}^{-3}$

Melting point: $318 \mathrm{~K}$

Mo $K \alpha$ radiation, $\lambda=0.71073 \AA$

Cell parameters from 3830 reflections

$\theta=3.3-27.6^{\circ}$

$\mu=0.07 \mathrm{~mm}^{-1}$

$T=120 \mathrm{~K}$

Plate, colourless

$0.20 \times 0.18 \times 0.01 \mathrm{~mm}$

Data collection

Bruker Nonius KappaCCD area-detector diffractometer

Radiation source: Bruker Nonius FR91 rotating anode

Graphite monochromator

Detector resolution: 9.091 pixels $\mathrm{mm}^{-1}$

$\varphi$ and $\omega$ scans

Absorption correction: multi-scan

(SADABS; Sheldrick, 2003)

$T_{\min }=0.987, T_{\max }=0.999$

17532 measured reflections

3830 independent reflections

1679 reflections with $I>2 \sigma(I)$

$R_{\text {int }}=0.150$

$\theta_{\text {max }}=27.6^{\circ}, \theta_{\min }=3.3^{\circ}$

$h=-126 \rightarrow 126$

$k=-6 \rightarrow 6$

$l=-9 \rightarrow 9$

Refinement

Refinement on $F^{2}$

Least-squares matrix: full

$R\left[F^{2}>2 \sigma\left(F^{2}\right)\right]=0.102$

$w R\left(F^{2}\right)=0.256$

$S=1.08$

3830 reflections

182 parameters

0 restraints

Primary atom site location: structure-invariant direct methods

Secondary atom site location: difference Fourier map

Hydrogen site location: inferred from

neighbouring sites

$\mathrm{H}$-atom parameters constrained

$w=1 /\left[\sigma^{2}\left(F_{\mathrm{o}}^{2}\right)+(0.0744 P)^{2}+7.8445 P\right]$

where $P=\left(F_{\mathrm{o}}^{2}+2 F_{\mathrm{c}}{ }^{2}\right) / 3$

$(\Delta / \sigma)_{\max }=0.001$

$\Delta \rho_{\max }=0.41 \mathrm{e}^{-3}$

$\Delta \rho_{\min }=-0.35$ e $\AA^{-3}$

Special details

Experimental. The scale factors in the experimental table are calculated from the 'size' command in the SHELXL97 input file. 
Fractional atomic coordinates and isotropic or equivalent isotropic displacement parameters $\left(\AA^{2}\right)$

\begin{tabular}{|c|c|c|c|c|}
\hline & $x$ & $y$ & $z$ & $U_{\text {iso }} * / U_{\text {eq }}$ \\
\hline $\mathrm{C} 1$ & $0.73264(3)$ & $0.7933(8)$ & $0.6134(6)$ & $0.0228(10)$ \\
\hline $\mathrm{O} 1$ & $0.74261(2)$ & $0.9565(6)$ & $0.6713(4)$ & $0.0309(8)$ \\
\hline $\mathrm{O} 2$ & $0.73386(2)$ & $0.6167(6)$ & $0.4964(4)$ & $0.0248(7)$ \\
\hline $\mathrm{C} 2$ & $0.71969(3)$ & $0.8502(8)$ & $0.7109(5)$ & $0.0201(10)$ \\
\hline $\mathrm{C} 3$ & 0.70703 & $0.7093(8)$ & $0.6268(6)$ & $0.0205(10)$ \\
\hline $\mathrm{C} 4$ & $0.69400(3)$ & $0.8111(8)$ & $0.7115(5)$ & $0.0189(9)$ \\
\hline $\mathrm{C} 5$ & $0.68092(3)$ & $0.6865(8)$ & $0.6249(6)$ & $0.0209(10)$ \\
\hline C6 & $0.66795(3)$ & $0.8041(8)$ & $0.7042(6)$ & $0.0192(9)$ \\
\hline $\mathrm{C} 7$ & $0.65479(3)$ & $0.6840(8)$ & $0.6155(6)$ & $0.0217(10)$ \\
\hline $\mathrm{C} 8$ & $0.64192(3)$ & $0.8070(8)$ & $0.6941(6)$ & $0.0217(10)$ \\
\hline C9 & $0.62897(3)$ & $0.6835(8)$ & $0.6108(5)$ & $0.0228(10)$ \\
\hline $\mathrm{C} 10$ & $0.61931(3)$ & $0.8140(8)$ & $0.5146(5)$ & $0.0218(10)$ \\
\hline C11 & $0.60645(3)$ & $0.6909(8)$ & $0.4322(6)$ & $0.0245(10)$ \\
\hline $\mathrm{C} 12$ & $0.59348(3)$ & $0.8106(8)$ & $0.5097(5)$ & $0.0193(9)$ \\
\hline $\mathrm{C} 13$ & $0.58035(3)$ & $0.6919(8)$ & $0.4225(6)$ & $0.0218(10)$ \\
\hline $\mathrm{C} 14$ & $0.56738(3)$ & $0.8113(8)$ & $0.4988(6)$ & $0.0208(10)$ \\
\hline $\mathrm{C} 15$ & 0.55429 & $0.6890(8)$ & $0.4087(6)$ & $0.0223(10)$ \\
\hline C16 & $0.54127(3)$ & $0.8076(8)$ & $0.4853(6)$ & $0.0224(10)$ \\
\hline $\mathrm{C} 17$ & $0.52827(3)$ & $0.6812(9)$ & $0.3945(6)$ & $0.0282(11)$ \\
\hline $\mathrm{C} 18$ & $0.51520(3)$ & $0.7981(9)$ & $0.4725(7)$ & $0.0360(12)$ \\
\hline $\mathrm{H} 1$ & 0.7506 & 0.9756 & 0.6267 & $0.046^{*}$ \\
\hline $\mathrm{H} 2 \mathrm{~A}$ & 0.7181 & 1.0481 & 0.7094 & $0.024 *$ \\
\hline $\mathrm{H} 2 \mathrm{~B}$ & 0.7210 & 0.7940 & 0.8428 & $0.024 *$ \\
\hline $\mathrm{H} 3 \mathrm{~A}$ & 0.7079 & 0.5117 & 0.6478 & $0.025^{*}$ \\
\hline H3B & 0.7065 & 0.7411 & 0.4906 & $0.025^{*}$ \\
\hline $\mathrm{H} 4 \mathrm{~A}$ & 0.6935 & 1.0102 & 0.6969 & $0.023 *$ \\
\hline H4B & 0.6945 & 0.7711 & 0.8467 & $0.023 *$ \\
\hline H5A & 0.6811 & 0.4884 & 0.6464 & $0.025^{*}$ \\
\hline H5B & 0.6807 & 0.7170 & 0.4885 & $0.025^{*}$ \\
\hline H6A & 0.6679 & 1.0026 & 0.6849 & $0.023^{*}$ \\
\hline H6B & 0.6681 & 0.7704 & 0.8402 & $0.023 *$ \\
\hline H7A & 0.6546 & 0.7151 & 0.4793 & $0.026^{*}$ \\
\hline H7B & 0.6548 & 0.4860 & 0.6367 & $0.026^{*}$ \\
\hline H8A & 0.6422 & 0.7806 & 0.8309 & $0.026^{*}$ \\
\hline H8B & 0.6418 & 1.0043 & 0.6697 & $0.026^{*}$ \\
\hline H9 & 0.6277 & 0.4949 & 0.6294 & $0.027^{*}$ \\
\hline H10 & 0.6206 & 1.0025 & 0.4956 & $0.026^{*}$ \\
\hline H11A & 0.6066 & 0.4935 & 0.4565 & $0.029 *$ \\
\hline H11B & 0.6062 & 0.7173 & 0.2955 & $0.029 *$ \\
\hline $\mathrm{H} 12 \mathrm{~A}$ & 0.5936 & 0.7795 & 0.6459 & $0.023 *$ \\
\hline H12B & 0.5935 & 1.0088 & 0.4888 & $0.023 *$ \\
\hline H13A & 0.5804 & 0.4938 & 0.4437 & $0.026^{*}$ \\
\hline H13B & 0.5802 & 0.7224 & 0.2862 & $0.026 *$ \\
\hline H14A & 0.5673 & 1.0094 & 0.4776 & $0.025 *$ \\
\hline H14B & 0.5675 & 0.7802 & 0.6350 & $0.025^{*}$ \\
\hline
\end{tabular}




$\begin{array}{lllll}\text { H15A } & 0.5542 & 0.7206 & 0.2725 & 0.027^{*} \\ \text { H15B } & 0.5544 & 0.4908 & 0.4295 & 0.027^{*} \\ \text { H16A } & 0.5411 & 1.0055 & 0.4635 & 0.027^{*} \\ \text { H16B } & 0.5414 & 0.7770 & 0.6216 & 0.027^{*} \\ \text { H17A } & 0.5282 & 0.7132 & 0.2583 & 0.034^{*} \\ \text { H17B } & 0.5285 & 0.4830 & 0.4152 & 0.034^{*} \\ \text { H18A } & 0.5151 & 0.7603 & 0.6063 & 0.054^{*} \\ \text { H18B } & 0.5073 & 0.7141 & 0.4086 & 0.054^{*} \\ \text { H18C } & 0.5149 & 0.9943 & 0.4521 & 0.054^{*}\end{array}$

Atomic displacement parameters $\left(\AA^{2}\right)$

\begin{tabular}{lllllll}
\hline & $U^{11}$ & $U^{22}$ & $U^{33}$ & $U^{12}$ & $U^{13}$ & $U^{23}$ \\
\hline C1 & $0.0142(16)$ & $0.022(2)$ & $0.032(3)$ & $0.0038(16)$ & $-0.0035(17)$ & $0.005(2)$ \\
O1 & $0.0171(11)$ & $0.0333(19)$ & $0.042(2)$ & $-0.0079(12)$ & $0.0022(12)$ & $-0.0123(15)$ \\
O2 & $0.0219(12)$ & $0.0276(18)$ & $0.0251(18)$ & $-0.0011(12)$ & $0.0008(11)$ & $-0.0047(15)$ \\
C2 & $0.0194(16)$ & $0.022(2)$ & $0.019(3)$ & $0.0035(16)$ & $-0.0021(15)$ & $-0.0017(19)$ \\
C3 & $0.0166(16)$ & $0.020(2)$ & $0.025(3)$ & $0.0031(15)$ & $-0.0015(16)$ & $0.0035(19)$ \\
C4 & $0.0191(16)$ & $0.019(2)$ & $0.019(2)$ & $0.0016(15)$ & $0.0015(16)$ & $-0.0005(18)$ \\
C5 & $0.0183(16)$ & $0.019(2)$ & $0.025(3)$ & $-0.0030(16)$ & $-0.0059(16)$ & $0.0025(19)$ \\
C6 & $0.0163(16)$ & $0.021(2)$ & $0.020(3)$ & $-0.0015(15)$ & $0.0024(15)$ & $0.0008(19)$ \\
C7 & $0.0201(16)$ & $0.025(2)$ & $0.020(3)$ & $-0.0024(16)$ & $-0.0050(16)$ & $0.0019(19)$ \\
C8 & $0.0194(17)$ & $0.028(2)$ & $0.018(3)$ & $-0.0018(16)$ & $0.0021(16)$ & $0.0018(19)$ \\
C9 & $0.0232(17)$ & $0.024(3)$ & $0.022(3)$ & $-0.0005(17)$ & $0.0028(17)$ & $-0.001(2)$ \\
C10 & $0.0154(16)$ & $0.023(2)$ & $0.026(3)$ & $-0.0026(16)$ & $0.0004(16)$ & $0.001(2)$ \\
C11 & $0.0197(17)$ & $0.026(2)$ & $0.027(3)$ & $-0.0010(16)$ & $-0.0068(17)$ & $0.001(2)$ \\
C12 & $0.0187(16)$ & $0.025(2)$ & $0.014(2)$ & $0.0003(16)$ & $-0.0002(15)$ & $0.0013(19)$ \\
C13 & $0.0193(17)$ & $0.026(2)$ & $0.020(3)$ & $-0.0018(16)$ & $-0.0023(16)$ & $0.0005(19)$ \\
C14 & $0.0209(17)$ & $0.021(2)$ & $0.020(3)$ & $0.0010(16)$ & $-0.0022(16)$ & $0.0010(19)$ \\
C15 & $0.0205(17)$ & $0.025(2)$ & $0.021(3)$ & $0.0001(16)$ & $-0.0032(16)$ & $-0.002(2)$ \\
C16 & $0.0232(17)$ & $0.021(2)$ & $0.023(3)$ & $0.0019(16)$ & $-0.0003(17)$ & $-0.0014(19)$ \\
C17 & $0.0207(17)$ & $0.037(3)$ & $0.027(3)$ & $-0.0025(17)$ & $-0.0039(17)$ & $0.001(2)$ \\
C18 & $0.0263(19)$ & $0.040(3)$ & $0.041(3)$ & $-0.0003(19)$ & $-0.0041(19)$ & $0.002(2)$ \\
& & & & & & \\
\hline
\end{tabular}

Geometric parameters $\left(\AA,{ }^{\circ}\right)$

\begin{tabular}{llll}
\hline $\mathrm{C} 1-\mathrm{O} 2$ & $1.221(5)$ & $\mathrm{C} 10-\mathrm{C} 11$ & $1.502(4)$ \\
$\mathrm{C} 1-\mathrm{O} 1$ & $1.323(4)$ & $\mathrm{C} 10-\mathrm{H} 10$ & 0.95 \\
$\mathrm{C} 1-\mathrm{C} 2$ & $1.508(5)$ & $\mathrm{C} 11-\mathrm{C} 12$ & $1.535(5)$ \\
$\mathrm{O} 1-\mathrm{H} 1$ & 0.8642 & $\mathrm{C} 11-\mathrm{H} 11 \mathrm{~A}$ & 0.99 \\
$\mathrm{C} 2-\mathrm{C} 3$ & $1.528(4)$ & $\mathrm{C} 11-\mathrm{H} 11 \mathrm{~B}$ & 0.99 \\
$\mathrm{C} 2-\mathrm{H} 2 \mathrm{~A}$ & 0.99 & $\mathrm{C} 12-\mathrm{C} 13$ & $1.527(4)$ \\
$\mathrm{C} 2-\mathrm{H} 2 \mathrm{~B}$ & 0.99 & $\mathrm{C} 12-\mathrm{H} 12 \mathrm{~A}$ & 0.99 \\
$\mathrm{C} 3-\mathrm{C} 4$ & $1.530(5)$ & $\mathrm{C} 12-\mathrm{H} 12 \mathrm{~B}$ & 0.99 \\
$\mathrm{C} 3-\mathrm{H} 3 \mathrm{~A}$ & 0.99 & $\mathrm{C} 13-\mathrm{C} 14$ & $1.530(5)$ \\
$\mathrm{C} 3-\mathrm{H} 3 \mathrm{~B}$ & 0.99 & $\mathrm{C} 13-\mathrm{H} 13 \mathrm{~A}$ & 0.99 \\
$\mathrm{C} 4-\mathrm{C} 5$ & $1.534(4)$ & $\mathrm{C} 13-\mathrm{H} 13 \mathrm{~B}$ & 0.99 \\
$\mathrm{C} 4-\mathrm{H} 4 \mathrm{~A}$ & 0.99 & $\mathrm{C} 14-\mathrm{C} 15$ & $1.540(4)$
\end{tabular}




\begin{tabular}{|c|c|c|c|}
\hline $\mathrm{C} 4-\mathrm{H} 4 \mathrm{~B}$ & 0.99 & $\mathrm{C} 14-\mathrm{H} 14 \mathrm{~A}$ & 0.99 \\
\hline $\mathrm{C} 5-\mathrm{C} 6$ & $1.536(5)$ & C14-H14B & 0.99 \\
\hline $\mathrm{C} 5-\mathrm{H} 5 \mathrm{~A}$ & 0.99 & $\mathrm{C} 15-\mathrm{C} 16$ & $1.534(5)$ \\
\hline $\mathrm{C} 5-\mathrm{H} 5 \mathrm{~B}$ & 0.99 & $\mathrm{C} 15-\mathrm{H} 15 \mathrm{~A}$ & 0.99 \\
\hline $\mathrm{C} 6-\mathrm{C} 7$ & $1.537(4)$ & $\mathrm{C} 15-\mathrm{H} 15 \mathrm{~B}$ & 0.99 \\
\hline C6-H6A & 0.99 & $\mathrm{C} 16-\mathrm{C} 17$ & $1.542(4)$ \\
\hline C6-H6B & 0.99 & $\mathrm{C} 16-\mathrm{H} 16 \mathrm{~A}$ & 0.99 \\
\hline $\mathrm{C} 7-\mathrm{C} 8$ & $1.536(5)$ & $\mathrm{C} 16-\mathrm{H} 16 \mathrm{~B}$ & 0.99 \\
\hline C7-H7A & 0.99 & $\mathrm{C} 17-\mathrm{C} 18$ & $1.540(5)$ \\
\hline C7-H7B & 0.99 & C17-H17A & 0.99 \\
\hline $\mathrm{C} 8-\mathrm{C} 9$ & $1.512(4)$ & C17-H17B & 0.99 \\
\hline $\mathrm{C} 8-\mathrm{H} 8 \mathrm{~A}$ & 0.99 & $\mathrm{C} 18-\mathrm{H} 18 \mathrm{~A}$ & 0.98 \\
\hline $\mathrm{C} 8-\mathrm{H} 8 \mathrm{~B}$ & 0.99 & $\mathrm{C} 18-\mathrm{H} 18 \mathrm{~B}$ & 0.98 \\
\hline $\mathrm{C} 9-\mathrm{C} 10$ & $1.318(5)$ & $\mathrm{C} 18-\mathrm{H} 18 \mathrm{C}$ & 0.98 \\
\hline $\mathrm{C} 9-\mathrm{H} 9$ & 0.95 & & \\
\hline $\mathrm{O} 2-\mathrm{C} 1-\mathrm{O} 1$ & $123.9(3)$ & $\mathrm{C} 9-\mathrm{C} 10-\mathrm{H} 10$ & 117.1 \\
\hline $\mathrm{O} 2-\mathrm{C} 1-\mathrm{C} 2$ & $124.3(3)$ & $\mathrm{C} 11-\mathrm{C} 10-\mathrm{H} 10$ & 117.1 \\
\hline $\mathrm{O} 1-\mathrm{C} 1-\mathrm{C} 2$ & $111.8(4)$ & $\mathrm{C} 10-\mathrm{C} 11-\mathrm{C} 12$ & $113.7(3)$ \\
\hline $\mathrm{C} 1-\mathrm{O} 1-\mathrm{H} 1$ & 128.4 & $\mathrm{C} 10-\mathrm{C} 11-\mathrm{H} 11 \mathrm{~A}$ & 108.8 \\
\hline $\mathrm{C} 1-\mathrm{C} 2-\mathrm{C} 3$ & $115.1(3)$ & $\mathrm{C} 12-\mathrm{C} 11-\mathrm{H} 11 \mathrm{~A}$ & 108.8 \\
\hline $\mathrm{C} 1-\mathrm{C} 2-\mathrm{H} 2 \mathrm{~A}$ & 108.5 & $\mathrm{C} 10-\mathrm{C} 11-\mathrm{H} 11 \mathrm{~B}$ & 108.8 \\
\hline $\mathrm{C} 3-\mathrm{C} 2-\mathrm{H} 2 \mathrm{~A}$ & 108.5 & $\mathrm{C} 12-\mathrm{C} 11-\mathrm{H} 11 \mathrm{~B}$ & 108.8 \\
\hline $\mathrm{C} 1-\mathrm{C} 2-\mathrm{H} 2 \mathrm{~B}$ & 108.5 & $\mathrm{H} 11 \mathrm{~A}-\mathrm{C} 11-\mathrm{H} 11 \mathrm{~B}$ & 107.7 \\
\hline $\mathrm{C} 3-\mathrm{C} 2-\mathrm{H} 2 \mathrm{~B}$ & 108.5 & $\mathrm{C} 13-\mathrm{C} 12-\mathrm{C} 11$ & $114.0(3)$ \\
\hline $\mathrm{H} 2 \mathrm{~A}-\mathrm{C} 2-\mathrm{H} 2 \mathrm{~B}$ & 107.5 & $\mathrm{C} 13-\mathrm{C} 12-\mathrm{H} 12 \mathrm{~A}$ & 108.8 \\
\hline $\mathrm{C} 2-\mathrm{C} 3-\mathrm{C} 4$ & $112.2(3)$ & $\mathrm{C} 11-\mathrm{C} 12-\mathrm{H} 12 \mathrm{~A}$ & 108.8 \\
\hline $\mathrm{C} 2-\mathrm{C} 3-\mathrm{H} 3 \mathrm{~A}$ & 109.2 & $\mathrm{C} 13-\mathrm{C} 12-\mathrm{H} 12 \mathrm{~B}$ & 108.8 \\
\hline $\mathrm{C} 4-\mathrm{C} 3-\mathrm{H} 3 \mathrm{~A}$ & 109.2 & $\mathrm{C} 11-\mathrm{C} 12-\mathrm{H} 12 \mathrm{~B}$ & 108.8 \\
\hline $\mathrm{C} 2-\mathrm{C} 3-\mathrm{H} 3 \mathrm{~B}$ & 109.2 & $\mathrm{H} 12 \mathrm{~A}-\mathrm{C} 12-\mathrm{H} 12 \mathrm{~B}$ & 107.7 \\
\hline $\mathrm{C} 4-\mathrm{C} 3-\mathrm{H} 3 \mathrm{~B}$ & 109.2 & $\mathrm{C} 12-\mathrm{C} 13-\mathrm{C} 14$ & $114.2(3)$ \\
\hline $\mathrm{H} 3 \mathrm{~A}-\mathrm{C} 3-\mathrm{H} 3 \mathrm{~B}$ & 107.9 & $\mathrm{C} 12-\mathrm{C} 13-\mathrm{H} 13 \mathrm{~A}$ & 108.7 \\
\hline $\mathrm{C} 3-\mathrm{C} 4-\mathrm{C} 5$ & $114.3(3)$ & $\mathrm{C} 14-\mathrm{C} 13-\mathrm{H} 13 \mathrm{~A}$ & 108.7 \\
\hline $\mathrm{C} 3-\mathrm{C} 4-\mathrm{H} 4 \mathrm{~A}$ & 108.7 & $\mathrm{C} 12-\mathrm{C} 13-\mathrm{H} 13 \mathrm{~B}$ & 108.7 \\
\hline $\mathrm{C} 5-\mathrm{C} 4-\mathrm{H} 4 \mathrm{~A}$ & 108.7 & $\mathrm{C} 14-\mathrm{C} 13-\mathrm{H} 13 \mathrm{~B}$ & 108.7 \\
\hline $\mathrm{C} 3-\mathrm{C} 4-\mathrm{H} 4 \mathrm{~B}$ & 108.7 & $\mathrm{H} 13 \mathrm{~A}-\mathrm{C} 13-\mathrm{H} 13 \mathrm{~B}$ & 107.6 \\
\hline $\mathrm{C} 5-\mathrm{C} 4-\mathrm{H} 4 \mathrm{~B}$ & 108.7 & $\mathrm{C} 13-\mathrm{C} 14-\mathrm{C} 15$ & $113.3(3)$ \\
\hline $\mathrm{H} 4 \mathrm{~A}-\mathrm{C} 4-\mathrm{H} 4 \mathrm{~B}$ & 107.6 & $\mathrm{C} 13-\mathrm{C} 14-\mathrm{H} 14 \mathrm{~A}$ & 108.9 \\
\hline $\mathrm{C} 4-\mathrm{C} 5-\mathrm{C} 6$ & $113.3(3)$ & $\mathrm{C} 15-\mathrm{C} 14-\mathrm{H} 14 \mathrm{~A}$ & 108.9 \\
\hline $\mathrm{C} 4-\mathrm{C} 5-\mathrm{H} 5 \mathrm{~A}$ & 108.9 & $\mathrm{C} 13-\mathrm{C} 14-\mathrm{H} 14 \mathrm{~B}$ & 108.9 \\
\hline $\mathrm{C} 6-\mathrm{C} 5-\mathrm{H} 5 \mathrm{~A}$ & 108.9 & $\mathrm{C} 15-\mathrm{C} 14-\mathrm{H} 14 \mathrm{~B}$ & 108.9 \\
\hline $\mathrm{C} 4-\mathrm{C} 5-\mathrm{H} 5 \mathrm{~B}$ & 108.9 & $\mathrm{H} 14 \mathrm{~A}-\mathrm{C} 14-\mathrm{H} 14 \mathrm{~B}$ & 107.7 \\
\hline $\mathrm{C} 6-\mathrm{C} 5-\mathrm{H} 5 \mathrm{~B}$ & 108.9 & $\mathrm{C} 16-\mathrm{C} 15-\mathrm{C} 14$ & $113.4(3)$ \\
\hline $\mathrm{H} 5 \mathrm{~A}-\mathrm{C} 5-\mathrm{H} 5 \mathrm{~B}$ & 107.7 & $\mathrm{C} 16-\mathrm{C} 15-\mathrm{H} 15 \mathrm{~A}$ & 108.9 \\
\hline $\mathrm{C} 5-\mathrm{C} 6-\mathrm{C} 7$ & $113.6(3)$ & $\mathrm{C} 14-\mathrm{C} 15-\mathrm{H} 15 \mathrm{~A}$ & 108.9 \\
\hline $\mathrm{C} 5-\mathrm{C} 6-\mathrm{H} 6 \mathrm{~A}$ & 108.8 & $\mathrm{C} 16-\mathrm{C} 15-\mathrm{H} 15 \mathrm{~B}$ & 108.9 \\
\hline $\mathrm{C} 7-\mathrm{C} 6-\mathrm{H} 6 \mathrm{~A}$ & 108.8 & $\mathrm{C} 14-\mathrm{C} 15-\mathrm{H} 15 \mathrm{~B}$ & 108.9 \\
\hline $\mathrm{C} 5-\mathrm{C} 6-\mathrm{H} 6 \mathrm{~B}$ & 108.8 & $\mathrm{H} 15 \mathrm{~A}-\mathrm{C} 15-\mathrm{H} 15 \mathrm{~B}$ & 107.7 \\
\hline
\end{tabular}




\begin{tabular}{|c|c|c|c|}
\hline $\mathrm{C} 7-\mathrm{C} 6-\mathrm{H} 6 \mathrm{~B}$ & 108.8 & $\mathrm{C} 15-\mathrm{C} 16-\mathrm{C} 17$ & $112.6(3)$ \\
\hline $\mathrm{H} 6 \mathrm{~A}-\mathrm{C} 6-\mathrm{H} 6 \mathrm{~B}$ & 107.7 & $\mathrm{C} 15-\mathrm{C} 16-\mathrm{H} 16 \mathrm{~A}$ & 109.1 \\
\hline $\mathrm{C} 8-\mathrm{C} 7-\mathrm{C} 6$ & $112.9(3)$ & $\mathrm{C} 17-\mathrm{C} 16-\mathrm{H} 16 \mathrm{~A}$ & 109.1 \\
\hline $\mathrm{C} 8-\mathrm{C} 7-\mathrm{H} 7 \mathrm{~A}$ & 109.0 & $\mathrm{C} 15-\mathrm{C} 16-\mathrm{H} 16 \mathrm{~B}$ & 109.1 \\
\hline $\mathrm{C} 6-\mathrm{C} 7-\mathrm{H} 7 \mathrm{~A}$ & 109.0 & $\mathrm{C} 17-\mathrm{C} 16-\mathrm{H} 16 \mathrm{~B}$ & 109.1 \\
\hline $\mathrm{C} 8-\mathrm{C} 7-\mathrm{H} 7 \mathrm{~B}$ & 109.0 & $\mathrm{H} 16 \mathrm{~A}-\mathrm{C} 16-\mathrm{H} 16 \mathrm{~B}$ & 107.8 \\
\hline $\mathrm{C} 6-\mathrm{C} 7-\mathrm{H} 7 \mathrm{~B}$ & 109.0 & $\mathrm{C} 18-\mathrm{C} 17-\mathrm{C} 16$ & $112.7(3)$ \\
\hline $\mathrm{H} 7 \mathrm{~A}-\mathrm{C} 7-\mathrm{H} 7 \mathrm{~B}$ & 107.8 & $\mathrm{C} 18-\mathrm{C} 17-\mathrm{H} 17 \mathrm{~A}$ & 109.0 \\
\hline $\mathrm{C} 9-\mathrm{C} 8-\mathrm{C} 7$ & $113.0(3)$ & $\mathrm{C} 16-\mathrm{C} 17-\mathrm{H} 17 \mathrm{~A}$ & 109.0 \\
\hline $\mathrm{C} 9-\mathrm{C} 8-\mathrm{H} 8 \mathrm{~A}$ & 109.0 & $\mathrm{C} 18-\mathrm{C} 17-\mathrm{H} 17 \mathrm{~B}$ & 109.0 \\
\hline $\mathrm{C} 7-\mathrm{C} 8-\mathrm{H} 8 \mathrm{~A}$ & 109.0 & $\mathrm{C} 16-\mathrm{C} 17-\mathrm{H} 17 \mathrm{~B}$ & 109.0 \\
\hline $\mathrm{C} 9-\mathrm{C} 8-\mathrm{H} 8 \mathrm{~B}$ & 109.0 & $\mathrm{H} 17 \mathrm{~A}-\mathrm{C} 17-\mathrm{H} 17 \mathrm{~B}$ & 107.8 \\
\hline $\mathrm{C} 7-\mathrm{C} 8-\mathrm{H} 8 \mathrm{~B}$ & 109.0 & $\mathrm{C} 17-\mathrm{C} 18-\mathrm{H} 18 \mathrm{~A}$ & 109.5 \\
\hline $\mathrm{H} 8 \mathrm{~A}-\mathrm{C} 8-\mathrm{H} 8 \mathrm{~B}$ & 107.8 & $\mathrm{C} 17-\mathrm{C} 18-\mathrm{H} 18 \mathrm{~B}$ & 109.5 \\
\hline $\mathrm{C} 10-\mathrm{C} 9-\mathrm{C} 8$ & $125.9(4)$ & $\mathrm{H} 18 \mathrm{~A}-\mathrm{C} 18-\mathrm{H} 18 \mathrm{~B}$ & 109.5 \\
\hline $\mathrm{C} 10-\mathrm{C} 9-\mathrm{H} 9$ & 117.1 & $\mathrm{C} 17-\mathrm{C} 18-\mathrm{H} 18 \mathrm{C}$ & 109.5 \\
\hline $\mathrm{C} 8-\mathrm{C} 9-\mathrm{H} 9$ & 117.1 & $\mathrm{H} 18 \mathrm{~A}-\mathrm{C} 18-\mathrm{H} 18 \mathrm{C}$ & 109.5 \\
\hline $\mathrm{C} 9-\mathrm{C} 10-\mathrm{C} 11$ & $125.7(4)$ & $\mathrm{H} 18 \mathrm{~B}-\mathrm{C} 18-\mathrm{H} 18 \mathrm{C}$ & 109.5 \\
\hline $\mathrm{O} 2-\mathrm{C} 1-\mathrm{C} 2-\mathrm{C} 3$ & $-11.8(6)$ & $\mathrm{C} 8-\mathrm{C} 9-\mathrm{C} 10-\mathrm{C} 11$ & $-179.9(4)$ \\
\hline $\mathrm{O} 1-\mathrm{C} 1-\mathrm{C} 2-\mathrm{C} 3$ & $168.8(3)$ & $\mathrm{C} 9-\mathrm{C} 10-\mathrm{C} 11-\mathrm{C} 12$ & $118.6(4)$ \\
\hline $\mathrm{C} 1-\mathrm{C} 2-\mathrm{C} 3-\mathrm{C} 4$ & $-170.7(3)$ & $\mathrm{C} 10-\mathrm{C} 11-\mathrm{C} 12-\mathrm{C} 13$ & $178.4(3)$ \\
\hline $\mathrm{C} 2-\mathrm{C} 3-\mathrm{C} 4-\mathrm{C} 5$ & $177.0(3)$ & $\mathrm{C} 11-\mathrm{C} 12-\mathrm{C} 13-\mathrm{C} 14$ & $-179.8(3)$ \\
\hline $\mathrm{C} 3-\mathrm{C} 4-\mathrm{C} 5-\mathrm{C} 6$ & $-176.6(3)$ & $\mathrm{C} 12-\mathrm{C} 13-\mathrm{C} 14-\mathrm{C} 15$ & $179.9(3)$ \\
\hline $\mathrm{C} 4-\mathrm{C} 5-\mathrm{C} 6-\mathrm{C} 7$ & $178.9(3)$ & $\mathrm{C} 13-\mathrm{C} 14-\mathrm{C} 15-\mathrm{C} 16$ & $179.8(3)$ \\
\hline $\mathrm{C} 5-\mathrm{C} 6-\mathrm{C} 7-\mathrm{C} 8$ & $-179.1(3)$ & $\mathrm{C} 14-\mathrm{C} 15-\mathrm{C} 16-\mathrm{C} 17$ & $-179.6(3)$ \\
\hline $\mathrm{C} 6-\mathrm{C} 7-\mathrm{C} 8-\mathrm{C} 9$ & $-178.4(3)$ & $\mathrm{C} 15-\mathrm{C} 16-\mathrm{C} 17-\mathrm{C} 18$ & $179.5(3)$ \\
\hline $\mathrm{C} 7-\mathrm{C} 8-\mathrm{C} 9-\mathrm{C} 10$ & $-118.8(4)$ & & \\
\hline
\end{tabular}

Hydrogen-bond geometry $\left(A,{ }^{\circ}\right)$

\begin{tabular}{lllll}
\hline$D-\mathrm{H} \cdots A$ & $D-\mathrm{H}$ & $\mathrm{H} \cdots A$ & $D \cdots A$ & $D-\mathrm{H} \cdots A$ \\
\hline $\mathrm{O} 1-\mathrm{H} 1 \cdots \mathrm{O} 2^{\mathrm{i}}$ & 0.87 & 1.86 & $2.684(3)$ & 158 \\
\hline
\end{tabular}

Symmetry code: (i) $-x+3 / 2,-y+3 / 2,-z+1$. 